\title{
Identification of an INSM1-binding site in the insulin promoter: negative regulation of the insulin gene transcription
}

\author{
Hong-Wei Wang ${ }^{1,2}$, Michelle Muguira ${ }^{1}$, Wei-Dong Liu ${ }^{1,2}$, Tao Zhang ${ }^{1,2}$, Chiachen Chen ${ }^{1}$, \\ Rebecca Aucoin ${ }^{1}$, Mary B Breslin ${ }^{1,2}$ and Michael S Lan ${ }^{1,2}$ \\ ${ }^{1}$ The Research Institute for Children, Children's Hospital, 200 Henry Clay Avenue, Research and Education Building, Room 2211, New Orleans, \\ Louisiana 70118, USA \\ ${ }^{2}$ Departments of Pediatrics and Genetics, Louisiana State University Health Sciences Center, New Orleans, Louisiana 70112, USA \\ (Correspondence should be addressed to M S Lan; Email: mlan@chnola-research.org) \\ (H-W Wang is now at Section of Endocrinology, School of Medicine, The University of Chicago, Chicago, Illinois 60637, USA)
}

\begin{abstract}
In this study, an insulinoma-associated antigen-1 (INSM1)binding site in the proximal promoter sequence of the insulin gene was identified. The co-transfection of INSM1 with rat insulin I/II promoter-driven reporter genes exhibited a 40-50\% inhibitory effect on the reporter activity. Mutational experiments were performed by introducing a substitution, GG to AT, into the INSM1 core binding site of the rat insulin I/II promoters. The mutated insulin promoter exhibited a three- to 20 -fold increase in the promoter activity over the wild-type promoter in several insulinoma cell lines. Moreover, INSM1 overexpression exhibited no inhibitory effect on the mutated insulin promoter. Chromatin immunoprecipitation assays using $\beta T C-1$, mouse fetal pancreas, and Ad-INSM1-transduced
\end{abstract}

human islets demonstrated that INSM1 occupies the endogenous insulin promoter sequence containing the INSM1-binding site in vivo. The binding of the INSM1 to the insulin promoter could suppress $\sim 50 \%$ of insulin message in human islets. The mechanism for transcriptional repression of the insulin gene by INSM1 is mediated through the recruitment of cyclin D1 and histone deacetylase-3 to the insulin promoter. Anti-INSM1 or anti-cyclin D1 morpholino treatment of fetal mouse pancreas enhances the insulin promoter activity. These data strongly support the view that INSM1 is a new zinc-finger transcription factor that modulates insulin gene transcription during early pancreas development.

Journal of Endocrinology (2008) 198, 29-39

\section{Introduction}

Insulin gene transcription is regulated by both the ubiquitous and the $\beta$-cell-specific transcription factors (Melloul et al. 2002, Brink 2003). In $\beta$-cells, multiple regulatory elements in the basal insulin promoter control insulin gene expression. These regulatory elements include E-box elements, A elements, the RIPE3b region, the $\mathrm{C} 1$ element, $\mathrm{G}$ elements, and some consensus binding sites such as Sp-1, CCAAT, and the cAMP regulatory element (CRE). A handful of transcription factors that govern $\beta$-cell differentiation ( $\mathrm{Pdx}-1, \mathrm{NeuroD} / \beta 2, \mathrm{Pax} 4 / 6$, and $N k x 2.2)$ have been shown to interact directly with the insulin promoter's regulatory elements. A majority of these factors not only bind to specific binding sites but also form multiprotein transcriptional complexes situated on the insulin promoter (Ohneda et al. 2000a, Qiu et al. 2002). The recruitment of the co-activator $\mathrm{p} 300$, along with the binding of Pdx-1 and bHLH factors, E47 and NeuroD/ $\beta 2$, in a complex with a high mobility group I protein on the E-A mini-enhancer, results in a synergistic activation of insulin gene expression (Ohneda et al. 2000b, Qiu et al. 2002, 2004, Mosley et al. 2004,
Stanojevic et al. 2004). Another A3/A4-binding homeoprotein, $\operatorname{lm} x 1.1$, activates transcription by acting synergistically, via its LIM domain, with E47 (German et al. 1992). Rat insulin promoter element 3 (RIPE3), which spans bases -125 to -86 , was shown to contain cell-specific and ubiquitous factors that confer enhancer activity (Shieh \& Tsai 1991). The C1/ RIPE3b1 (-118/-107 bp) binding factor regulates both pancreatic $\beta$-cell-specific and glucose-regulated transcription of the insulin gene, which includes members of the large Maf transcription factor family (Kataoka et al. 2002, Olbrot et al. 2002, Matsuoka et al. 2003, Zhao et al. 2005). By investigating novel regulatory elements on the rat insulin promoter, a conserved sequence spanning between -61 to $-50 \mathrm{bp}$ and resembling the binding site of insulinoma-associated antigen-1 (INSM1, formerly IA-1) was identified (Breslin et al. 2002).

The INSM1 gene encodes a $58 \mathrm{kDa}$ protein that contains five zinc-finger DNA-binding motifs, originally isolated from a human insulinoma subtraction library (Goto et al. 1992). The expression pattern is restricted to the fetal pancreas, fetal nervous system, and in tumors of neuroendocrine origin (Xie et al. 2002, Zhu et al. 2002, Breslin et al. 2003). 
Functional studies have revealed that the amino terminus of the INSM1 protein possesses repressor activity and that the zinc-finger motifs recognize the conserved target sequence, $\mathrm{T}^{\mathrm{G}} / \mathrm{T}^{\mathrm{C}} / \mathrm{T}^{\mathrm{C}} / \mathrm{T}^{\mathrm{T}} / \mathrm{A}_{\mathrm{A}} \mathrm{GGG}^{\mathrm{G}} / \mathrm{T}_{\mathrm{T}}^{\mathrm{G}} / \mathrm{A}$, which is located in the promoter regions of both the NeuroD/ $\beta 2$ and INSM1 genes (Breslin et al. 2002, Liu et al. 2006). In vitro induction of AR42J amphicrine cells and normal human ductal epithelial cells induction into insulin-producing cells suggested that INSM1's gene expression is closely associated with the expression of isletspecific transcription factors and it was shown to be an immediate downstream the target gene of neurogenin3 (Ngn3; Zhu et al. 2002, Mellitzer et al. 2006, Breslin et al. 2007). Global INSM1 mutant mice revealed that INSM1 plays an essential role for the development of both pancreatic $\beta$-cells and intestinal endocrine cells (Gierl et al. 2006).

An INSM1-binding site in the insulin promoter region was identified in this study, suggesting that insulin could be a target gene of the INSM1. This INSM1-binding site is highly conserved among humans, rats, and mice. EMSA and reporter gene transfection analyses of rat and mouse insulin promoters (MIP) support that the INSM1 protein binds and suppresses insulin promoter activity. The occupancy of the INSM1 transcription factor on the insulin promoter sequence was confirmed by the chromatin immunoprecipitation (ChIP) assay. It was also shown that overexpression of INSM1 in human islets could directly downregulate the insulin gene message, whereas INSM1 anti-sense treatment of embryonic mouse pancreas increased insulin promoter activity. These results strongly support its negative regulatory role in insulin gene transcription. Furthermore, the mechanism for transcriptional repression of the insulin gene by INSM1 is mediated through the recruitment of cyclin D1 and histone deacetylase-3 (HDAC-3) to the insulin promoter.

\section{Materials and Methods}

\section{Cell culture and reagents}

Insulinoma (MIN6, HIT, and RIN) and HeLa cell lines were obtained from the American Type Culture Collection (Manasas, VA, USA) and maintained as described by the protocol. $\beta$ TC-1 cells were kindly provided by Dr E H Leiter (Jackson Laboratory, Bar Harbor, ME, USA). The mouse or rabbit anti-cyclin D1, anti-HDAC-3, and anti-Flag antisera were obtained from Biosource (Camarillo, CA, USA), Upstate (Lake Placid, NY, USA), and Sigma respectively. A mouse anti-INSM1 monoclonal antibody (6-1-1) was generated against the carboxyl-terminal peptide of the INSM1 protein. Recombinant adenoviral vectors were constructed as described (Liu et al. 2006).

\section{EMSA}

EMSA was performed using two copies of double-strand oligo spanning the -64 to $-47 \mathrm{bp}$ region of the rat insulin I promoter (containing INSM1-binding site),
5'-AAAGTCCAGGGGGCAGAG- $3^{\prime}$, and the complementary strand. The double-stranded oligo was endlabeled using $\left[\gamma^{32} \mathrm{P}\right] \mathrm{ATP} \quad(3000 \mathrm{Ci} / \mathrm{mmol}$, Perkin Elmer Life Sciences) and T4 polynucleotide kinase (New England Biolabs, Beverly, MA, USA). The INSM1 carboxyl-terminal peptide (Cpep, aa 257-510) was synthesized using a pGBKT7-Cpep vector and the TNT coupled rabbit reticulocyte lysate kit (Promega). The synthesis of Cpep was confirmed by western blot analysis using an anti-Cpep antibody (6-1-1). Nuclear extracts from cultured cell lines were prepared as described (Dignam et al. 1983). The EMSA reaction condition was described previously (Liu et al. 2006). Competitive EMSA was performed using unlabeled cold probe, random probe, or mutant probe.

\section{Construction of rat insulin I and II promoter mutants}

The rat insulin I ( -410 to $+50 \mathrm{bp}$; accession number, J00747) and insulin II promoters $(-792$ to $-4 \mathrm{bp}$; accession number, J00748) were PCR amplified from rat genomic DNA. The amplified sequences were confirmed by sequence analysis and cloned into a pGL3-Basic vector (Promega) for promoterdriven luciferase assay. The INSM1-binding site on the

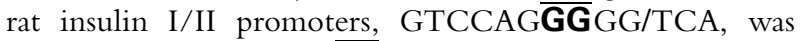
mutated into GTCCAG $\overline{\mathbf{A T}}$ GG/TCA using PCR primers, PInI-1, GATCGGTACCTGAGCTAAGAATCCA; PInI-2, GATCAGATCTACATACCTGCTTGCT; PInI-3, CGGCAAAGTCCAGATGGCAGAGAGGA; PInI-4, TCCTCTCTCTGCCATCTGGACTTTGCCG; PInII-1, GATCGGTACCCCCAACCACTCCAAGTGG; PInII-2, GATCCTCGAGGGTTACTGAATCCCAC; PInII-3, AAACAGCAAAGTCCAGATGTCAGGGG; and PInII-4, CCCCTGACTACTGGACTTTGCTGTTT. The mutant rat insulin I and II promoters were confirmed by sequence analysis and subcloned into the pGL3-Basic vector for promoter-driven luciferase assays. Three copies of the INSM1-binding site derived from the insulin promoter were subcloned in front of the E1bTATA basic promoter and the luciferase gene.

\section{Transient transfection and insulin promoter assays}

The rat insulin promoter driven luciferase vector $(1.0 \mu \mathrm{g})$, rInIp or rInIIp (I: $-410 /+50$ bp; II: $-792 /-4$ bp), was transfected into a $70 \%$ confluent rat insulinoma cell line, RIN, in the presence or absence of CMV-INSM1 (pcDNA3INSM1) $(0 \cdot 5 \mu \mathrm{g})$. An empty pcDNA3 vector was also included to ensure that each transfection had an equal amount of DNA. A CMV- $\beta$-galactosidase construct $(0 \cdot 5 \mu \mathrm{g})$ was used as an internal control to normalize transfection efficiency. Twenty-four hours post-transfection, the cells were washed with PBS and collected for luciferase and $\beta$-galactosidase assays (Promega). Each experiment was performed at least six times, and the results represent the average of six times \pm s.E.M. For rat insulin I/II promoter mutants (rInIpm and rInIIpm), three insulinoma cell lines, MIN (mouse), RIN (rat), and HIT (hamster), and HeLa 
cells were used. The wild-type rat insulin I/II promoter activities were designated as $100 \%$ and the mutant promoter activities were expressed as fold relative to wild-type activity. Each experiment was performed at least six times, and the results represent the average of six times \pm s.E.M.

\section{Co-immunoprecipitation and western blot analysis}

Insulinoma cell line, $\beta \mathrm{TC}-1$, was transduced with Flag-tag Ad-INSM1 (m.o.i. 50:1). The transduced cells were harvested $48 \mathrm{~h}$ post-transduction. Cell lysates were prepared with a protein-binding buffer $(20 \mathrm{mM}$ Tris- $\mathrm{Cl}(\mathrm{pH} 7 \cdot 5), 150 \mathrm{mM}$ $\mathrm{NaCl}, 0 \cdot 5 \% \mathrm{NP}-40,0 \cdot 1 \%$ Triton X-100, 1 mM phenylmethylsulfonyl fluoride). For co-immunoprecipitation, $300 \mu \mathrm{g}$ cell lysates were pre-cleared with rProtein G-Agarose, and incubated for $2 \mathrm{~h}$ at $4{ }^{\circ} \mathrm{C}$ with anti-cyclin D1, anti-Flag, or normal control IgG antibody, followed by incubation for $1 \mathrm{~h}$ at $4{ }^{\circ} \mathrm{C}$ with rProtein G-Agarose beads. The beads were washed $5 \times$ with a binding buffer, separated on $10 \%$ SDS-PAGE, and transblotted onto a nitrocellulose membrane (Invitrogen). INSM1, cyclin D1, and HDAC-3 were detected by western blotting (1:1000) with each antibody. The horseradish peroxidase (HRP)-conjugated anti-rabbit or anti-mouse secondary antibody (1:4000) and chemiluminescent substrate (Pierce, Rockford, IL, USA) was used for detection.

\section{ChIP assay}

ChIP assays were performed in $\beta$ TC- 1 , mouse fetal pancreas, and Ad-INSM1 transduced human islets. A ChIP assay kit (Upstate) was used and the procedures were followed as described (Liu et al. 2006). A mouse monoclonal antibody (6-1-1) to INSM1, anti-HDAC-3 (Biosource), anti-Flag (Sigma), and anti-acetyl-H3/H4 antibodies (Upstate) were used in ChIP assays. The PCR primers used to detect target sequences were as follows: mouse insulin II (forward) 5'-ACCTAGCACCAGGGAAGTGTTT-3'; (reverse) 5'-ATAGACCAAAGCACCTCCTCT-3', (132 bp); human insulin (forward) $5^{\prime}$-TGAGGAAGAGGTGCTGACGA-3'; (reverse) 5'-CCTCTTCTGATGCAGCCTGT-3' (225 bp); and phosphoenolpyruvate carboxykinase (PCK) (forward) 5'-GAGTGACACCTCACAGCTGTGG-3'; (reverse) $5^{\prime}$-GGCAGGCCTTTGGATCATAGCC-3' (339 bp) used as a control.

Northern blot and real-time RT-PCR analysis of Ad-INSM1transduced human islets

Fresh human islets (5000 IEQs, 95\% purity) obtained from Islet Cell Resources (City of Hope SC-ICR Center, Duarte, CA, USA) was transduced with either Ad-INSM1 (100:1 m.o.i.) or Ad-LacZ (100:1 m.o.i.) for 48 h. Total RNA was isolated using Trizol reagent (Invitrogen). Total RNA $(20 \mu \mathrm{g})$ was separated on a formaldehyde denaturing gel, transblotted, and subjected to northern blot analysis. INSM1 and glyceraldehyde-3-phosphate dehydrogenase probes were random primer labeled using $\boldsymbol{\alpha}_{-}{ }^{32} \mathrm{P}-\mathrm{dCTP}$ (Perkin-Elmer Life Sciences, Waltham, MA, USA). The same blot was stripped and re-hybridized with each probe. The insulin message was determined by quantitative realtime RT-PCR. First-strand cDNA was prepared from $5 \mu \mathrm{g}$ total RNA with the SuperScript II RT kit (Invitrogen). One-tenth of the cDNA was used as a template with IQ SYBRgreen supermix (Bio-Rad) for PCR. The expression level of the insulin gene was corrected for that of the $\beta$-actin housekeeping gene. The human insulin primers are as follows: (forward) 5'-CCTTGTGAACCAACACCTG-3', (reverse) 5'-GTAGAAGAAGCCTCGTTCCC- $3^{\prime}$ (80 bp) and human $\beta$-actin primers are (forward) 5'-CTGTACGCCAACACAGT GCT-3', (reverse) 5'-GCTCAGGAGGAGCAATGATC-3' (127 bp). Three batches of human islets were received from ICR for these studies.

\section{Morpholino treatment of MIP-enhanced green fluorescence protein} (MIP-EGFP) fetal pancreas

Fetal pancreata were harvested from E16 day MIP-EGFP transgenic mice obtained from the Jackson Laboratory (Hara et al. 2003). Each fetal pancreas was cultured in Costar 96-well black opaque plate. The culture was incubated in DMEM (10\% FBS and penicillin/streptomycin) supplemented with INSM1 anti-sense (5'-GCATGTTGGCGCGGTGAAAAGGGCG-3'), cyclin D1 anti-sense (5'-GCAGCACAGGAGCTGGTGTTCCATG-3'), or inverted INSM1 control (5'-GCGGGAAAAGTGGCGCGGTTGTACG-3') morpholino oligonucleotides $(25 \mu \mathrm{M})$ (Gene Tools, LLC) and partial medium changes were performed after $24 \mathrm{~h}$. The fluorescence signal was read at different time points using a BIO-TEK fluorimeter at excitation 485/20 and emission 528/20. The experiments were repeated three times using embryos from different pregnant mice.

\section{Statistical analysis}

Statistical analysis was performed using the Student's $t$-test for unpaired comparisons. Data are presented as means \pm s.E.M.; $P<0 \cdot 05$ was considered significant.

\section{Results}

INSM1 functions as a transcriptional repressor of the insulin gene

The INSM1 protein consists of 510 amino acid residues with five zinc-finger DNA-binding motifs at the carboxyl terminus (Goto et al. 1992). A selected and amplified binding site selection strategy was used to identify the INSM1 consensus DNAbinding site, $\mathrm{T}^{\mathrm{G}} / \mathrm{T}^{\mathrm{C}} / \mathrm{T}^{\mathrm{C}} / \mathrm{T}^{\mathrm{T}} / \mathrm{A} G \mathrm{GGG}^{\mathrm{G}} / \mathrm{T}^{\mathrm{G}} / \mathrm{A}$ (Fig. 1) (Breslin et al. 2002). By searching the eukaryotic promoter GenBank database, a potential INSM1-binding site was found at the proximal region of the insulin gene promoter. This potential INSM1-binding site is highly conserved in human, rat, and mouse insulin genes (Fig. 1). In order to determine whether the 


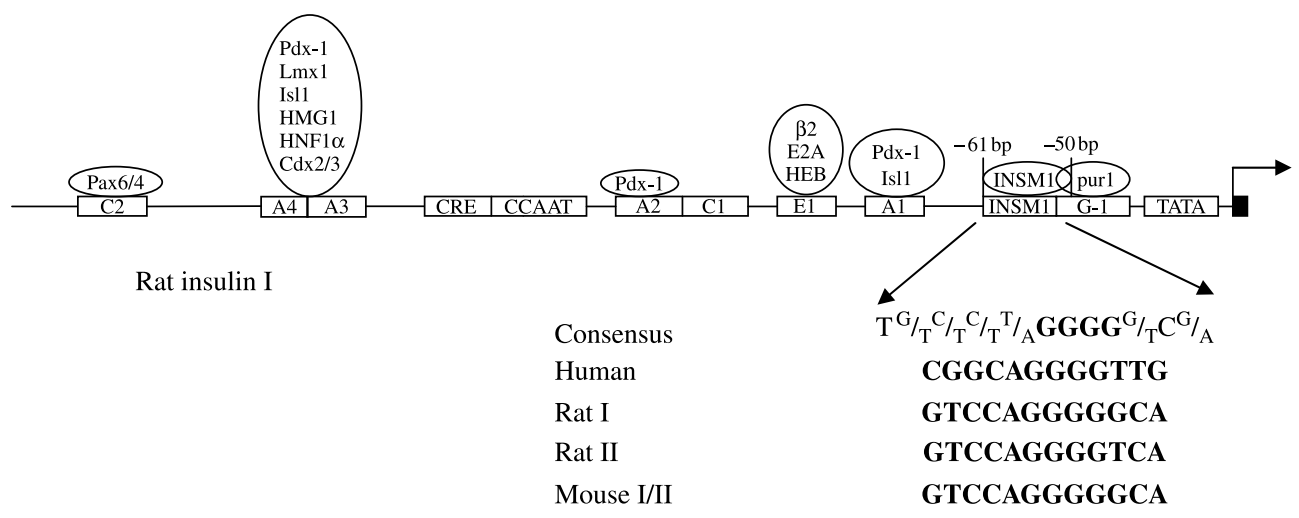

Rat insulin I promoter: ${ }^{-64}$ AAAGTCCAGGGGGCAGAG ${ }^{-47}$
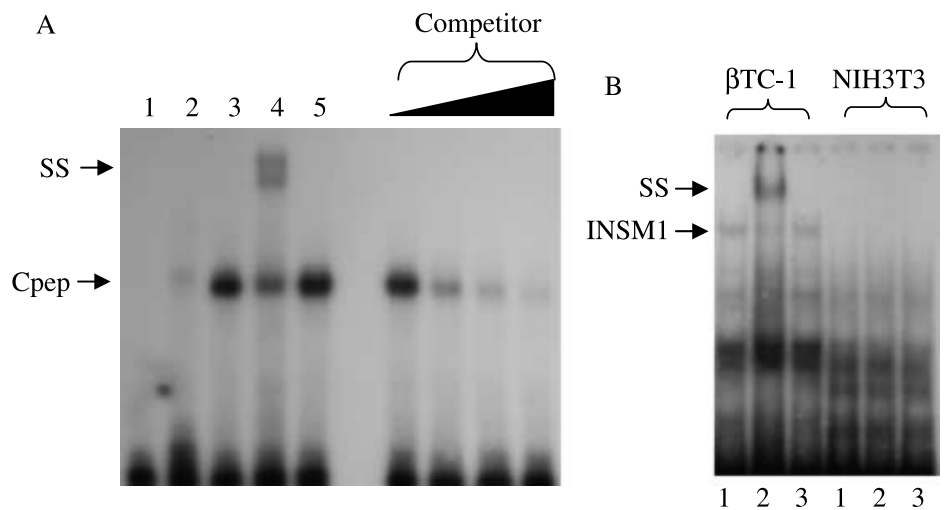

Figure 1 EMSA. The INSM1 consensus binding site sequence was derived from our previous study (Breslin et al. 2002). The conserved INSM1-binding sequence in the insulin gene is located the proximal promoter region of humans, mice, and rats. A modified schematic of the rat insulin I gene promoter with major regulatory elements and a subset of binding factors was derived from a previously published chart (Ohneda et al. 2000a). A tandem repeated double-strand oligo spanning the -64 to -47 bp region of the rat insulin I promoter was end-labeled as a probe. The carboxyl terminus (Cpep) of INSM1 was in vitro transcribed and translated in a TNT coupled rabbit reticulocyte lysate system. (A) The probe alone (lane 1), and probe with control lysate (lane 2), Cpep lysate (lane 3), Cpep lysate plus anti-Cpep antibody (lane 4) and Cpep lysate plus mouse IgG control (lane 5) are shown in the left panel. The right-hand panel shows different concentrations of cold competitor oligos $(0-200 \times)$.

(B) The same probe was gel-shifted with nuclear extracts $(10 \mu \mathrm{g})$ derived from $\beta$-TC1 and NIH3T3 cells (lane 1), with anti-Cpep antibody (lane 2), and with control mouse IgG (lane 3). Arrows indicated gel-shift bands and super-shift (SS) bands in both translated and INSM1-positive nuclear extracts.

INSM1 protein can bind to the INSM1-binding site within the insulin gene promoter, we used an in vitro synthesized carboxyl terminus of INSM1 (Cpep, 257-510 amino acid residues) protein or nuclear extracts isolated from insulinoma cells (INSM1-positive) or NIH3T3 (INSM1-negative), and a probe $(-47 /-64 \mathrm{bp})$ spanning the rat insulin I promoter region in an EMSA study. As shown in Fig. 1A, a band shift was seen with the Cpep lysate. This band was super-shifted by an INSM1 antibody, but not with the normal IgG control demonstrating a specific protein-DNA interaction. Competition experiments demonstrated that the binding of the Cpep lysate and insulin promoter probe can be abolished by increasing the unlabeled cold probe concentration. A similar EMSA using the nuclear extracts isolated from insulinomas and NIH3T3 cells indicated that a unique shifted band and super-shifted band can also be seen with the nuclear extracts from the INSM1-positive cell line $\beta$ TC-1, but not with the INSM1-negative NIH3T3 cell line (Fig. 1B). Taken together, our data support that INSM1 protein is capable of binding to the insulin promoter. To further determine whether INSM1 functions as a transcriptional repressor of the insulin gene, rat insulin promoters I $(-410 /+50 \mathrm{bp})$ and II $(-792 /-4 \mathrm{bp})$ were subcloned into a pGL3-Basic vector. These insulin promoter-driven luciferase vectors were transfected into RIN cells with or without an INSM1 expression vector. As shown in Fig. 2A, INSM1 exhibits a $40-50 \%$ repression effect on either rat insulin I or II promoter activity. To further investigate whether this inhibitory effect was mediated through the INSM1-binding site within the insulin promoter, three copies of the INSM1-binding site sequence derived from rat insulin I or mouse insulin I/II 
A
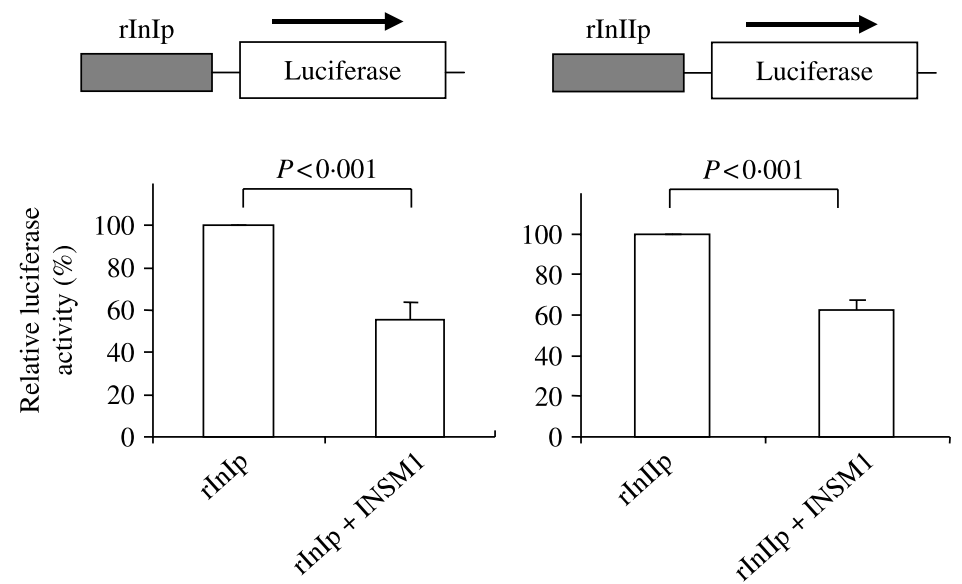

B
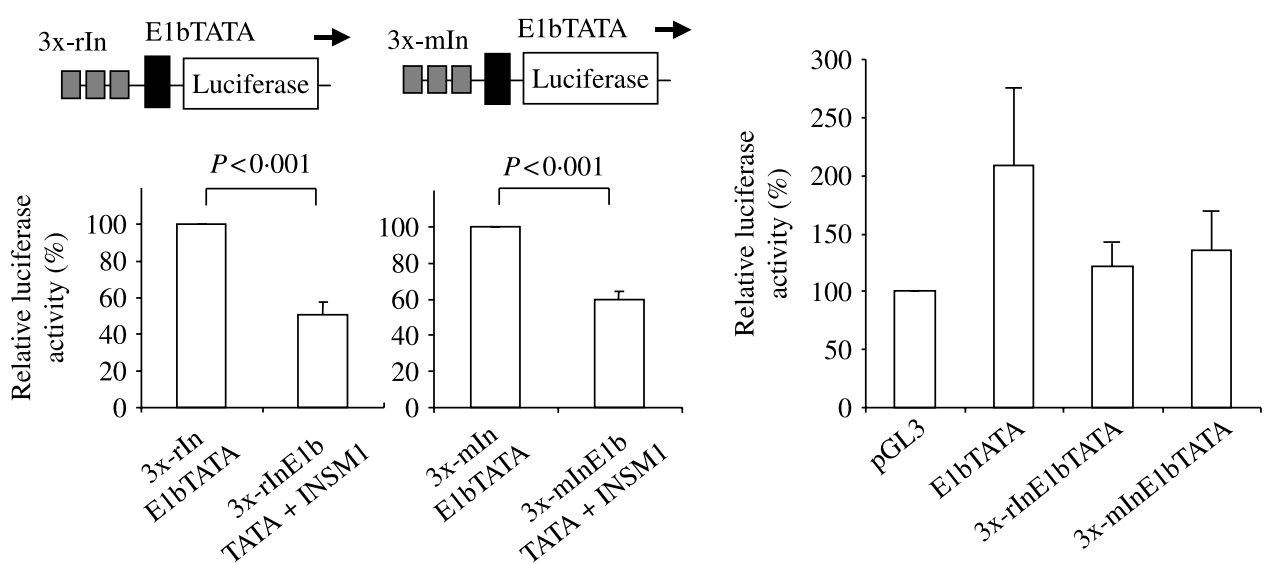

Figure 2 INSM1 suppresses mouse and rat insulin promoter activity. (A) Rat insulin I (rInlp: -410 to $+50 \mathrm{bp}$ ) and insulin II promoters (rlnllp: -792 to $-4 \mathrm{bp}$ ) were subcloned into a pGL3-Basic vector for luciferase activity assay. Insulin promoter activity was shown by co-transfection of reporter construct with INSM1 expression vector or control pcDNA3 vector into RIN cells. (B) The same experimental procedures were used for the (In) $)_{3}$-E1bTATA reporter construct to measure suppressor activity. Three copies of INSM1binding site-E1bTATA reporter vector were compared with E1bTATA-reporter or pGL3-Basic vector. The experiments were repeated at least six times and normalized with $\beta$-gal.

promoters were subcloned into an E1bTATA-luciferase vector. The transfection of these vectors into RIN cells with or without an INSM1 expressing vector resulted in a $40-50 \%$ repression (Fig. 2B). While comparing three-copy binding site-E1bTATA with E1bTATA alone showed slight decreases in their reporter activities suggesting that endogenous INSM1 imposed a suppression effect but no other activators affecting the same binding sequence. These results demonstrated that the INSM1binding site is involved in the INSM1-mediated repression activity.

Our previous study showed that mutation analysis of any two $G$ nucleotides in the GGGG core consensus binding site abolished INSM1 binding, suggesting that the core GGGG nucleotides are critical for INSM1-binding activity (Breslin et al. 2002). In the present study, the middle GG bases within the core GGGG of the rat insulin I/II INSM1-binding site were mutated into AT. The wild-type promoter luciferase or the mutated promoter luciferase vector was transfected into different insulinoma cell lines or HeLa cells. In each cell line, when compared with the wild-type rat insulin I promoter activity, the mutated rat insulin I promoter activity increased eight- to 20-fold in insulinoma cell lines, but not in HeLa cells (Fig. 3A). Similarly, the mutated rat insulin II promoter activity increased two- to threefold in insulinoma cell lines with no change observed in the HeLa cells (Fig. 3A). The drastic differences of wild-type rat insulin promoter I and II versus mutant promoters could be due to the additional sequence present in both the promoters. Further co-transfection studies revealed that overexpressed INSM1 lost its suppressor effect on the mutated rat insulin I promoter in RIN cells (Fig. 3B), indicating that the core 'GGGG' within the INSM1-binding site is critical for transcriptional repression activity. An EMSA competitive study using either random probe or mutant probe also demonstrated no 
A
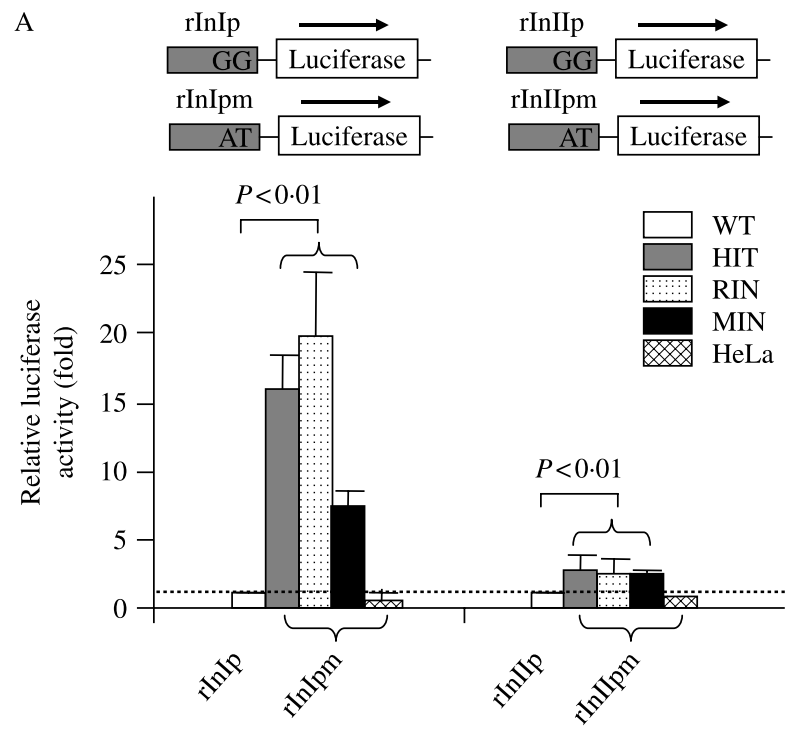

B

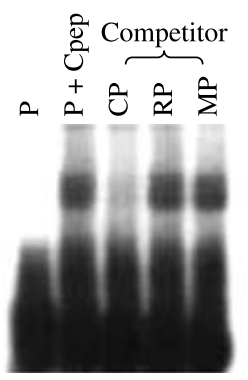

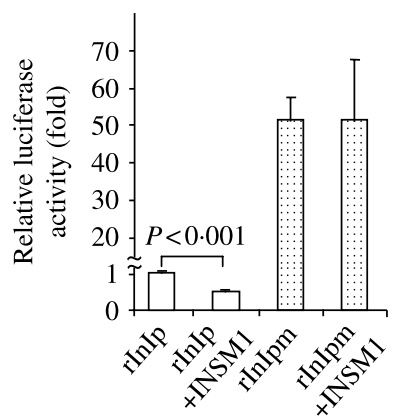

Figure 3 The INSM1-binding site in the insulin promoter is a negative regulatory element. The INSM1-binding site in the rat insulin I/II promoter, GTCCAG $\mathbf{G G G G / T C A , ~ w a s ~ m u t a t e d ~ i n t o ~}$ GTCCAGATGG/TCA. The mutant rat insulin I and II promoters (rInlpm and rlnllpm) were confirmed by sequence analysis and subcloned into the pGL3-Basic vector for promoter driven luciferase assay. (A) Three insulinoma cell lines and a HeLa cell line were tested for wild-type and mutant promoter activities. The mutant promoter activities were compared with wild-type promoter activities in each individual cell line. (B) The mutated rat insulin I promoter activity was examined for its response to the INSM1 suppression effect. INSM1 suppresses wild-type rat insulin I promoter $(P<0 \cdot 001)$, but not the mutant rat insulin I promoter in RIN cells. Transfections were normalized with $\beta$-gal. Each cell line was repeated at least six times. An EMSA study using cold probe $(\mathrm{CP})$, random probe (RP), or mutant probe (MP) to compete with gelshift band (arrow) show that mutant probe is no longer capable of competing with wild-type binding sequence.

competition for the shift band (Fig. 3B). Collectively, these results strongly support that INSM1 is a transcriptional repressor of the insulin gene.

\section{INSM1 downregulates endogenous insulin gene expression}

ChIP assay is designed to study protein-DNA interactions in vivo. In the previous section, we demonstrated in vitro that INSM1 binds and represses the rat insulin I/II promoter region containing an INSM1-binding site (Fig. 2). Here, ChIP assays were performed to investigate the occupancy of the mouse insulin gene promoter region by endogenous INSM1 in $\beta$ TC- 1 cells. $\beta$ TC- 1 cells were cross-linked with formaldehyde, sheared, and precipitated with anti-INSM1. As shown in Fig. 4, the mouse insulin II promoter sequence $(-152 /-21 \mathrm{bp})$ was selectively amplified by PCR from chromatin precipitated by anti-INSM1 antibody, but not from the chromatin precipitated by normal mouse IgG. A PCK promoter sequence was included to show the lack of INSM1 binding as a negative control. These experiments confirmed that endogenous INSM1 occupies the endogenous mouse insulin gene promoter region spanning the INSM1binding site in $\beta T C-1$ cells. Since INSM1 is not expressed in the human adult islets, we used the Ad-INSM1-transduced human islets to show that the INSM1 protein is capable of binding to the human insulin gene promoter by ChIP assay (Fig. 4B). To demonstrate that INSM1 is actually capable of suppressing insulin gene expression, the suppression effect of INSM1 on endogenous insulin gene expression was tested by using an adenovirus overexpression system in normal human islets. Fresh human islets were transduced with Ad-LacZ or Ad-INSM1 for $48 \mathrm{~h}$. Northern blot analysis showed a strong expression of the Ad-INSM1 message in contrast to the absence of endogenous INSM1. Quantitative real-time RT-PCR analyses revealed that the insulin message was suppressed by $\sim 50 \%$ in the INSM1 overexpressing islets (Fig. 4C). The suppression effect seen in the human islets is consistent with the transient transfection study using the rat insulin promoter. The suppression effect of INSM1 should change the acetylation pattern of chromatin on the insulin promoter. We further performed the ChIP assay on the Ad-INSM1 or Ad-LacZ-transduced human islets using antiacetyl H3/H4. As shown in Fig. 4D, acetyl-H4 on the insulin promoter decreased in Ad-INSM1-transduced islets suggesting that a suppression event could occur. Since INSM1 functions as a developmental transcription factor in fetal pancreas, we performed a ChIP assay using E16 day fetal pancreas and included fetal liver as a negative control. Figure 6A shows that fetal pancreas expressing INSM1 occupies the insulin promoter in contrast to the fetal liver control.

INSM1 transcriptional repression activity is mediated through the recruitment of cyclin D1 and $\mathrm{HDAC-3}$

INSM1 was shown to form a transcriptional complex with cyclin D1 and HDAC-3 (Liu et al. 2006). The molecular mechanisms of INSM1 repressor activity on the insulin gene promoter were investigated by transient transfection and co-immunoprecipitation of INSM1 and cyclin D1 in $\beta$ TC-1 insulinoma cells. Figure 5A shows that the overexpression of cyclin D1 could enhance the INSM1 repression effect on the three copies of the INSM1-binding site derived from the rat insulin I promoter linked to the E1bTATA basic promoter. However, only cyclin D1 or HDAC-3 with the reporter 
A

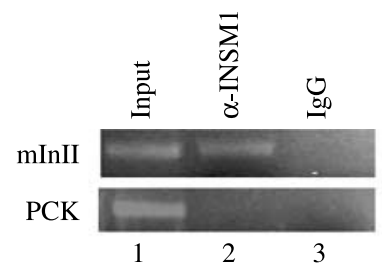

B
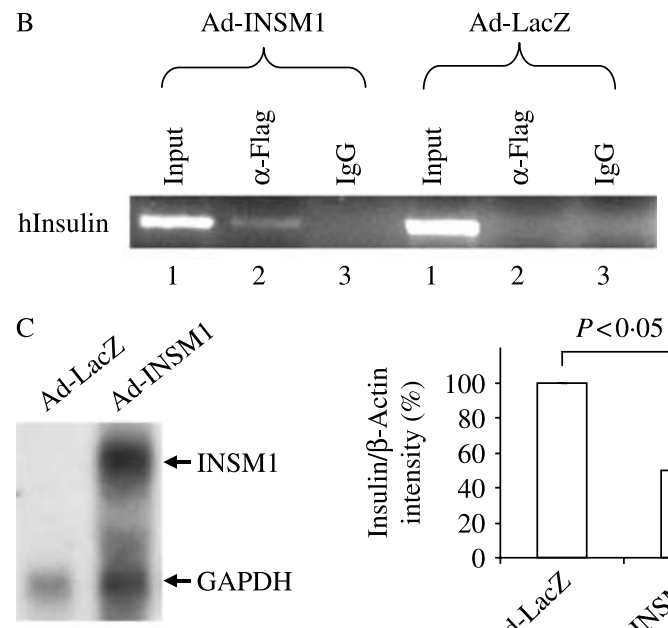

$\mathrm{D}$
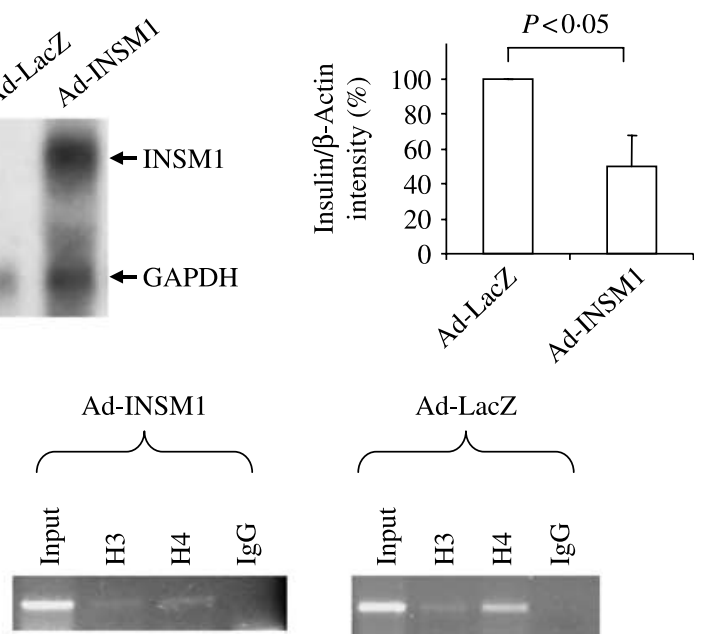

Figure 4 INSM1 binds to the endogenous insulin promoter and downregulates insulin gene transcription. (A) ChIP assay in $\beta$-TC1. Formaldehyde cross-linked chromatin from $\beta$-TC1 cells was incubated with an antibody to INSM1 (lane 2). Immunoprecipitated DNA was analyzed by PCR with primers specific to the transcriptional regulatory sequences of the mouse insulin II promoter, containing the INSM1-binding site and a control promoter sequence derived from PCK. As controls, PCRs were performed with input DNA (lane 1) and DNA immunoprecipitated by normal IgG (lane 3). (B) ChIP assay in human islets. Fresh human islets obtained from ICR were transduced with Ad-LacZ (5000 IEQs) or Ad-Flag-INSM1 (5000 IEQs) for $48 \mathrm{~h}$. A ChIP assay similar to that above was performed using anti-Flag antibody (lane 2) or normal control IgG (lane 3). The human insulin promoter sequence was amplified from both input DNA (lane 1) and Ad-Flag-INSM1transduced islets. No signal was detected in the control IgG or Ad-LacZ transduced islets. (C) Northern analysis and real-time RT-PCR. Fresh human islets obtained from ICR were transduced with Ad-LacZ (5000 IEQs) or Ad-INSM1 (5000 IEQs) for $48 \mathrm{~h}$. Total RNA was isolated using Trizol reagent. Twenty micrograms of RNA were separated on a denaturing agarose gel and hybridized with

${ }^{32}$ P-labeled INSM1 and GAPDH probes sequentially. In the righthand panel, quantitative real-time RT-PCR was performed with $\beta$ actin as an internal control. The graph displays the insulin message after normalization with $\beta$-actin from three different experiments. (D) ChIP analysis was performed with the Ad-LacZ- or Ad-INSM1transduced human islets with anti-acetyl $\mathrm{H} 3$ or $\mathrm{H} 4$ antibody. Each ChIP experiment was repeated to ensure its reproducibility. vector showed a minor suppression effect probably due to the endogenous INSM1. The enhanced suppression effect by the overexpression of INSM1 and cyclin D1 is statistically significant, but the low magnitude of enhancement could be explained by the presence of endogenous cyclin D1. We also compared the effects of INSM1, cyclin D1, and HDAC-3 in $(\mathrm{In})_{3}$-E1bTATA and mutant (In) ${ }_{3}$-E1bTATA reporter vectors (Fig. 5B). The wild-type promoter showed a more than $60 \%$ suppression by the INSM1-cyclin D1-HDAC-3 complex in contrast to that of the mutant promoter, which was unaffected. Figure 5C shows that overexpressed INSM1 and endogenous cyclin D1 or histone deacetylase (HDAC-3) are capable of interacting with each other using the co-immunoprecipitation method. Furthermore, anti-cyclin D1 is capable of co-precipitating HDAC-3 directly. In ChIP experiment, anti-HDAC-3 antibody successfully precipitated the endogenous HDAC-3-insulin II promoter complex, suggesting that HDAC-3 could bind to the INSM1 transcription factor to regulate insulin promoter activity (Fig. 5D). These data illustrate that INSM1, cyclin D1, and HDAC-3 constitute three important components for the transcriptional repression of the insulin gene.

\section{INSM1 regulates insulin promoter in the embryonic mouse pancreas}

To further clarify that INSM1 is truly involved in regulating insulin promoter activity during pancreas development, we selected a MIP-EGFP transgenic model (Hara et al. 2003). This animal model expresses the EGFP reporter under the control of mouse insulin promoter. Day E16 fetal pancreata were isolated from mice expressing MIP-EGFP. Explants were incubated with INSM1 or cyclin D1-specific anti-sense morpholino oligos to reduce INSM1 or cyclin D1 expression. An invert INSM1 oligo was used as a negative control. The anti-sense oligonucleotide technology to knock down specific gene expression in embryonic pancreas in vitro has been reported previously (Prasadan et al. 2002, Li et al. 2004, Mellitzer et al. 2006). Our study revealed that during the first $14 \mathrm{~h}$ of incubation, all three groups of fetal pancreas, control, anti-INSM1, and anti-cyclin D1 exhibited a burst of fluorescence signals (Fig. 6B). The control morpholino treatment showed no further increase at 24 and $38 \mathrm{~h}$. By contrast, anti-INSM1 treatment showed a similar burst of fluorescence at $14 \mathrm{~h}$ and continued to increase steadily through the $38 \mathrm{~h}$ incubation. The gradual increase in the insulin promoter activity suggested that anti-INSM1 morpholino treatment alleviates the INSM1 suppression effect to the insulin promoter. Anti-cyclin D1 treatment presented a lower burst of fluorescence signal at $14 \mathrm{~h}$ incubation. However, a steady increase in the fluorescence signal was observed through $38 \mathrm{~h}$. It is speculated that anticyclin D1 could not only affect the insulin promoter, but also affect the pancreas growth in general. Therefore, these results demonstrated that INSM1 and cyclin D1 are involved in insulin promoter suppression during pancreas development. 
A

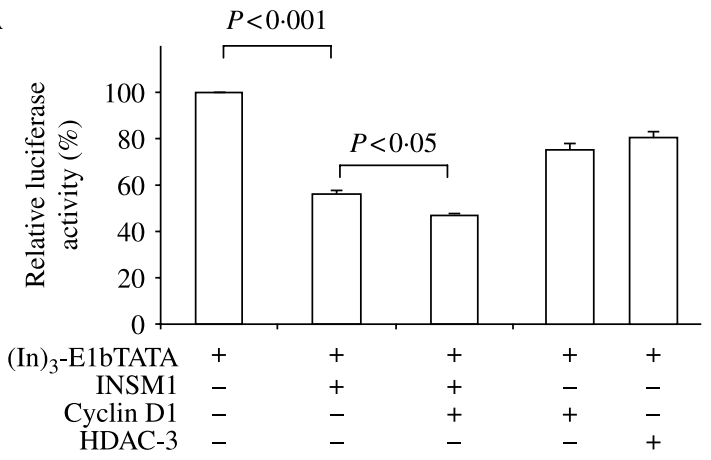

$\mathrm{C}$

$$
\overbrace{\alpha \text {-Flag- }}^{\text {IP }}
$$

WB IgG INSM1

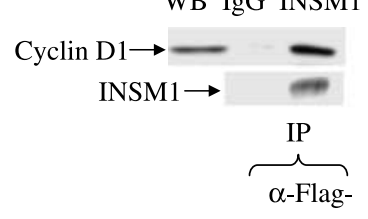

WB IgG INSM1

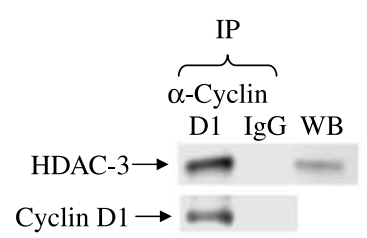

Cyclin D1 $\rightarrow$

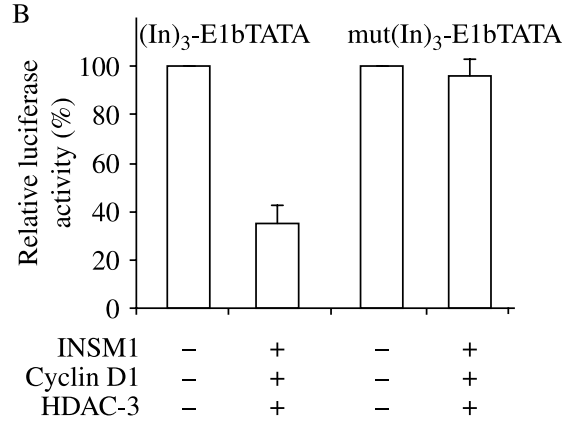

$\mathrm{D}$

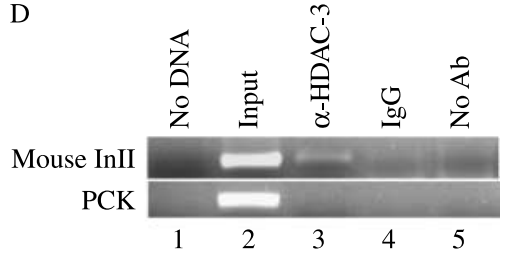

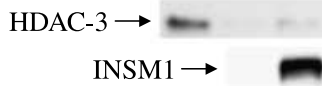

Figure 5 Cyclin D1-mediated HDAC-3 recruitment contributes to insulin transcriptional repression. (A) Cyclin D1 cooperates with INSM1 to suppress the rat INSM1-binding site-E1 bTATA reporter vector. Cyclin D1 or HDAC3 alone exhibits minor suppression effect probably due to the endogenous INSM1 in $\beta$ TC-1 cells. (B) (In) $3^{-}$ E1bTATA and mutant (In) $)_{3}$-E1bTATA reporter vectors were co-transfected with INSM1, cyclin D1, and HDAC-3. The wild-type promoter showed a greater than $60 \%$ of suppression by INSM1-cyclin D1-HDAC-3 complex in contrast to that of the mutant promoter, which showed little suppression effect. (C) Co-immunoprecipitation of INSM1, cyclin D1, and HDAC-3. $\beta$ TC-1 cells were transduced with Ad-INSM1 for $48 \mathrm{~h}$. Cell lysates were immunoprecipitated with anti-Flag-INSM1, anti-cyclin D1, or normal IgG control antiserum. Co-precipitated HDAC-3 was revealed by western blot analysis with an anti-HDAC-3 antibody. The precipitates were also blotted for cyclin D1 or Flag-INSM1 to ensure their presence in the immunoprecipitated complex. (D) HDAC-3 is associated with the insulin gene promoter sequence in vivo by ChIPassay. Formaldehyde cross-linked chromatin isolated from Ad-INSM1-transduced $\beta$-TC1 cells were incubated with rabbit antiserum to HDAC-3 (lane 3) or control normal IgG (lane 4). Immunoprecipitated DNA was analyzed by PCR with primers specific for the transcriptional regulatory sequence of the mouse insulin II promoter (the same primers used in Fig. 4A) and the PCK promoter sequence. As controls, PCRs were performed with no DNA (lane 1), input DNA (lane 2), DNA immunoprecipitated by normal IgG (lane 4), and DNA that was immunoprecipitated in the absence of antiserum (lane 5). The ChIP assay was repeated in two separate experiments.

\section{Discussion}

$\beta$-Cell-specific expression of the insulin gene is regulated by a complex network consisting of cis-acting elements and transregulators. The most extensively studied enhancer/promoter region in the insulin gene is within $350 \mathrm{bp}$ upstream from the transcriptional start site (Ohneda et al. 2000a, Melloul et al. 2002). This region is highly conserved in humans and rodents, suggesting that they share common regulatory mechanisms to regulate insulin gene transcription. Among these elements, $\mathrm{A}, \mathrm{C}$, and $\mathrm{E}$ are considered to be the major determinants of $\beta$ cell-specific expression of the insulin gene. Transcription factors Pdx1, NeuroD/ $\beta 2 / E 47$, and MafA bind to the A-E minienhancer/promoter cooperatively with the co-activator p300, and activate insulin gene transcription
(Qiu et al. 2002, 2004, Mosley et al. 2004, Stanojevic et al. 2004, Zhao et al. 2005). In contrast to the positive regulation of insulin gene expression, negative regulation was not as frequently reported. Nkx2.2 is an islet-enriched transcription factor belonging to the NK2 family. Gene targeting experiments have shown that $\mathrm{Nkx} 2.2$ is important for islet cell development and function, especially for the maturation of $\beta$-cells (Sussel et al. 1998). A recent study demonstrated that $\mathrm{Nkx} 2.2$ bound to the rat insulin II promoter region $(-140 /-119 \mathrm{bp})$ both in vitro and in vivo, and inhibited insulin promoter activity (Cissell et al. 2003). Another transcription factor, Pax-4, was shown to downregulate the human insulin promoter through a C2 element located between -253 and -244 bp (Campbell et al. 1999). In the present study, an INSM1 zinc-finger transcription factor 
A
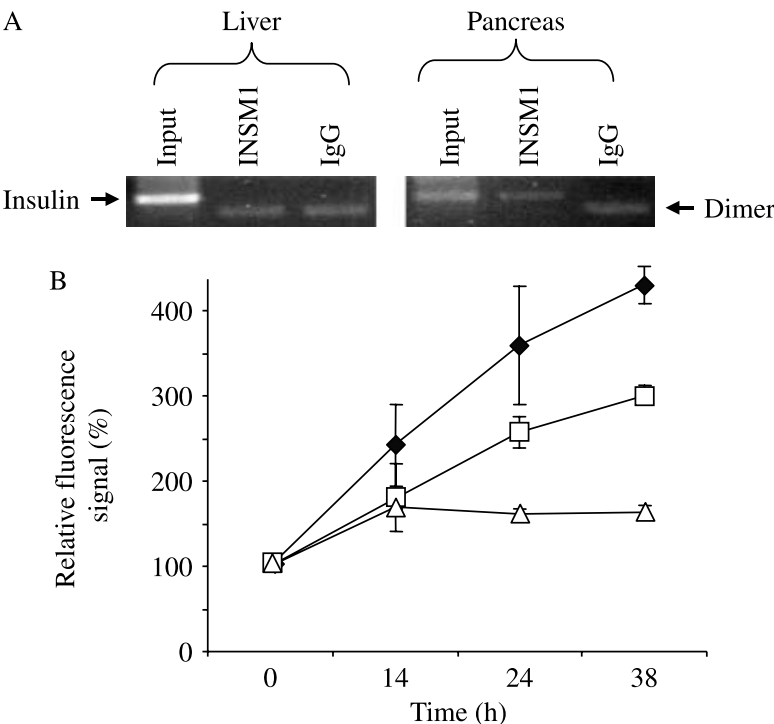

Figure 6 INSM1 interacts with insulin promoter in fetal pancreas. (A) Fetal pancreas ChIP assay was performed using E16 day fetal pancreas or fetal liver. Due to the small amount of DNA sample, we increased the PCR cycle number to 45 . The rest of the procedures were followed as above. Lower band labeled as dimer represents primer dimer from PCR. (B) E16 day fetal pancreas was isolated from MIP-EGFP transgenic mouse. Fetal pancreata incubated in a 96-well plate was supplemented with either anti-INSM1 (close diamond), anti-cyclin D1 (open square), or control oligo (open triangle) morpholinos. At different time points, fluorescence signals were measured using BIO-TEK fluorimeter. Relative fluorescence signal was calculated against the 0 -h time point. The results were shown as the average of three different experiments.

binding site was identified at the proximal promoter region $(-61 /-50 \mathrm{bp})$ of the rat insulin gene, which is highly conserved between the human, rat, and mouse species. It is located between the A1 and G1 elements and is in very close proximity to the TATA box-binding site. Gel mobility-shift analysis and transient transfection assays determined the physical interaction and repression by INSM1. The mutation of GGGG to GATG within the INSM1-binding site greatly increased rat insulin I promoter activity and modestly increased the rat insulin II promoter activity. The same mutation resulted in the loss of INSM1 transcriptional repressor activity in transfected cells, supporting the notion that INSM1 binds to the predicted INSM1 sequence. In addition to the in vitro studies, ChIP assays support the finding that the endogenous INSM1 protein is capable of binding to the mouse insulin II promoter sequence. The overexpression of Ad-INSM1 in normal human islets represses the insulin message by $\sim 50 \%$. To further address the question whether the INSM1 suppression effect to the insulin promoter is physiologically significant, we demonstrated the occupancy of INSM1 on the insulin promoter in E16 day fetal pancreas. We also employed anti-sense experiments to an MIP-EGFPtagged embryonic mouse pancreas. The knock down of INSM1 showed a steady increase in green fluorescence signals. Thus, INSM1 is truly involved in insulin promoter suppression during pancreas development.

The transient expression patterns of INSM1 in the fetal pancreas were reported in both humans and mice (Zhu et al. 2002, Mellitzer et al. 2006). Since the INSM1 gene is silenced in the adult stage of the pancreas, it suggests that INSM1 plays a minimum role in the insulin gene regulation in mature $\beta$-cells. However, due to its close association with ngn3, NeuroD/ $\beta 2$, and insulin genes, its biological function cannot be ignored during the early stages of embryonic pancreatic endocrine cell development. Recently, INSM1 has been shown to be essential for pancreatic endocrine development (Gierl et al. 2006). During pancreas development, we and others have shown that INSM1 is an immediate downstream target gene of ngn3 (Mellitzer et al. 2006, Breslin et al. 2007). The differentiation of adult ductal epithelial cells by ngn3 clearly indicated that ngn3 first activates the INSM1 gene and subsequently activates NeuroD/ $\beta 2$. Our previous studies have shown that the NeuroD/ $\beta 2$ and $\mathrm{E} 47$ heterodimer activate the INSM1 gene (Breslin et al. 2003). Conversely, INSM1 can suppress NeuroD/ $/ 2$ gene expression (Liu et al. 2006). NeuroD/ $\beta 2$ is a basic helix-loop-helix transcription factor that plays an essential role in $\beta$-cell development and insulin gene activation (Naya et al. 1995, 1997). These studies situate INSM1 in between ngn3 and NeuroD/B2 in pancreatic endocrine cell differentiation, generating a scenario in which ngn3 initiates the transcription regulatory cascade by first activating INSM1 gene expression, and subsequently activating the NeuroD/ $\beta 2$ gene (Huang et al. 2000, Mellitzer et al. 2006). When ngn 3 expression becomes silenced, NeuroD/ $\beta 2$ continues to activate INSM1 gene expression contrary to its feedback inhibitory mechanism (Breslin et al. 2003). Therefore, INSM1 might play a pivotal role in the control of NeuroD/ $\beta 2$ and insulin gene expression at an early stage of endocrine cell development. Although it is unclear whether insulin gene expression during pancreas development contributes to endocrine cell differentiation, it is speculated that in the presence of INSM1 in the early stages of endocrine cell differentiation, the suppression of insulin gene transcription in premature islets could be an important event in islet maturation. Mellitzer et al. (2006) observed that knock down of the INSM1 gene in E12.5 dorsal pancreatic epithelia blocks islet cell differentiation, and results in a $40 \%$ decrease in $\alpha$ - and $\beta$-cells, whereas the overexpression of ngn 3 and INSM1 in pancreatic duct cells can enhance NeuroD/ $\beta 2$ and Pax-4 gene expression as compared with ngn 3 alone. The discrepancy between decreasing the $\alpha$ - and $\beta$-cells versus increasing the insulin promoter activity through morpholino treatment could be explained by the fact that our study is directly measuring insulin gene expression instead of $\alpha$ - and $\beta$-cell differentiation. It is most likely that either reduction or enhancement of INSM1 gene expression could disturb normal pancreatic endocrine cell differentiation, which subsequently affects mature islet cell formation. Consistently, INSM1 gene ablation results in a dramatic decrease in pancreatic $\beta$-cells (Gierl et al. 2006). All of these observations suggest that INSM1 should 
contribute greatly to pancreatic endocrine cell development. Overall, INSM1 not only plays a role as a transient negative regulator of NeuroD/ $\beta 2$, insulin, and its own gene expression, but is also required for endocrine cell differentiation.

The primary function of cyclin D1 is to initiate cell cycle progression by association with cyclin-dependent kinase 4 (CDK4) resulting in the phosphorylation of the $\mathrm{Rb}$ protein. Phosphorylation of the $\mathrm{Rb}$ protein disrupts its association with E2F, allowing transcriptional activation of S-phase genes. Additionally, cyclin D1 displays CDK4-independent roles in transcriptional regulation (Coqueret 2002). Cyclin D1 inhibits the transcriptional activation of multiple transcription factors and nuclear receptors (Skaper et al. 1996, Ganter et al. 1998, Inoue \& Sherr 1998, Adnane et al. 1999, Knudsen et al. 1999, Bienvenu et al. 2001, Lin et al. 2002, Ratineau et al. 2002, Fu et al. 2005a). The co-repressor activities of cyclin D1 were shown by us and others to be closely associated with the recruitment of HDACs (Lin et al. 2002, Fu et al. 2005a, Liu et al. 2006). By contrast, cyclin D1 could also display inhibition to transcriptional activation by association with the histone acetyltransferase p300 (Ratineau et al. 2002, Fu et al. $2005 b$ ). In our previous studies, we have demonstrated that INSM1 interacts with cyclin D1 to recruit HDACs to form a transcriptional repressor complex on the NeuroD/ $\beta 2$ gene (Liu et al. 2006). It is conceivable that INSM1 exercises its repression effect upon the insulin gene through a similar mechanism. We performed transient transfection, co-immunoprecipitation, and ChIP assays in insulinoma cells to demonstrate that cyclin D1 cooperates with INSM1 to enhance its repression effect, and that INSM1 is capable of immunoprecipitating endogenous cyclin D1 and HDAC-3. Furthermore, anti-HDAC-3 antibody precipitates the transcriptional complex with the mouse insulin II promoter sequence in $\beta \mathrm{TC}-1$ cells. A transcriptional complex of INSM1-cyclin D1-HDAC-3 is situated on the insulin gene promoter to confer the repression effect.

In summary, a unique INSM1-binding site in the insulin promoter that downregulated insulin gene transcription, was identified. The repression effect of INSM1 is mediated through the recruitment of cyclin D1 and HDAC-3. These studies demonstrate that INSM1 could be a negative regulator for insulin gene during early endocrine pancreas development.

\section{Declaration of Interest}

The authors declare that there is no conflict of interest that would prejudice the impartiality of this scientific work.

\section{Funding}

This work was supported by funds from the Research Institute for Children, Children's Hospital at New Orleans, and a grant from the NIDDK, National Institutes of Health, DK61436 (to M S L).

\section{Acknowledgements}

We thank Dr C Q Cai's technical support. We are grateful to the 'ICR Basic Science Islet Distribution Program' for providing fresh human islets for this study.

\section{References}

Adnane J, Shao Z \& Robbins PD 1999 Cyclin D1 associates with the TBPassociated factor TAFII250 to regulate Sp1-mediated transcription. Oncogene 18 239-247.

Bienvenu F, Gasean H \& Coqueret O 2001 Cyclin D1 represses STAT3 activation through a CDK4-independent mechanism. Journal of Biological Chemistry 276 16840-16847.

Breslin MB, Zhu M, Notkins AL \& Lan MS 2002 Neuroendocrine differentiation factor, IA-1, is a transcriptional repressor and contains a specific DNA-binding domain: identification of consensus IA-1 binding sequence. Nucleic Acids Research 30 1038-1045.

Breslin MB, Zhu M \& Lan MS 2003 NeuroD1/E47 regulates the E-box element of a novel zinc-finger transcription factor, IA-1, in developing nervous system. Journal of Biological Chemistry 278 38991-38997.

Breslin MB, Wang HW, Pierce A, Aucoin R \& Lan MS 2007 Neurogenin 3 recruits $\mathrm{CBP}$ co-activator to facilitate histone $\mathrm{H} 3 / \mathrm{H} 4$ acetylation in the target gene INSM1. FEBS Letters 581 949-954.

Brink C 2003 Promoter elements in endocrine pancreas development and hormone regulation. Cellular and Molecular Life Sciences 60 1033-1048.

Campbell SC, Cragg H, Elrick LJ, Macfarlane WM, Shennan KIJ \& Docherty K 1999 Inhibitory effect of Pax4 on the human insulin and islet amyloid polypeptide (IAPP) promoters. FEBS Letters 463 53-57.

Cissell MA, Zhao L, Sussel L, Henderson E \& Stein R 2003 Transcription factor occupancy of the insulin gene in vivo. Journal of Biological Chemistry 278 751-756.

Coqueret O 2002 Linking cyclins to transcriptional control. Gene 299 35-55.

Dignam JD, Lebovitz RM \& Roeder RG 1983 Accurate transcription initiation by RNA polymeraseII in a soluable extract from isolated mammalian nuclei. Nucleic Acids Research 11 1475-1489.

Fu M, Rao M, Bouras T, Wang C, Wu K, Zhang X, Li Z, Yao TP \& Pestell RG $2005 a$ Cyclin D1 inhibits PPARr-meidated adipogenesis through HDAC recruitment. Journal of Biological Chemistry 280 16934-16941.

Fu M, Wang C, Rao M, Wu X, Bouras T, Zhang X, Li Z, Jiao X, Yang J, Li A et al. 2005 b Cyclin D1 represses p300 transactivation through a cyclindependent kinase-independent mechanism. Journal of Biological Chemistry 280 29728-29742.

Ganter B, Fu S \& Lipsick JS 1998 D-type cyclins repress transcriptional activation by the v-Myb but not the c-Myb DNA-binding domain. EMBO Journal 17 255-268.

German MS, Wang J, Chadwick RB \& Rutter WJ 1992 Synergistic activation of the insulin gene by a LIM-homeodomain protein and a basic helix-loophelix protein: building a functional insulin minienhancer complex. Genes and Development 6 2165-2176.

Gierl MS, Karoulias N, Wende H, Strehle M \& Birchmeier C 2006 The Zincfinger factor Insm1 (IA-1) is essential for the development of pancreatic beta cells and intestinal endocrine cells. Genes and Development 20 2465-2478.

Goto Y, DeSilva MG, Toscani A, Prabhakar BS, Notkins AL \& Lan MS 1992 A novel human insulinoma-associated cDNA, IA-1, encodes a protein with zinc-finger DNA-binding motifs. Journal of Biological Chemistry 267 15252-15257.

Hara M, Wang X, Kawamura T, Bindokas VP, Dizon RF, Alcoser SY, Magnuson MA \& Bell GI 2003 Transgenic mice with green fluorescent protein-labeled pancreatic beta cells. American Journal of Physiology. Endocrinology and Metabolism 284 E177-E183.

Huang HP, Liu M, El-hodiri HM, Chu K, Jamrich M \& Tsai MJ 2000 Regulation of the pancreatic islet-specific gene beta2 (neruoD) by neurogenin 3. Molecular and Cellular Biology 20 3292-3307. 
Inoue K \& Sherr CJ 1998 Gene expression and cell cycle arrest mediated by transcription factor DMP1 is antagonized by D-type cyclins through a cyclin-dependent kinase indenpendent mechanism. Molecular and Cellular Biology 18 1590-1600.

Kataoka K, Han S, Shioda S, Hirai M, Nishizawa M \& Handa H 2002 MafA is a glucose-regulated and pancreatic beta-cell specific transcriptional activator for the insulin gene. Journal of Biological Chemistry 277 49903-49910.

Knudsen KE, Cavence WK \& Arden KC 1999 D-type cyclins complex with the androgen receptor and inhibit its transcriptional transactivation ability. Cancer Research 59 2297-2301.

Li Z, Manna P, Kobayashi H, Spilde T, Bhatia A, Preuett B, Prasadan K, Hembree M \& Gittes GK 2004 Multifaceted pancreatic mesenchymal control of epithelial lineage selection. Developmental Biology 269 252-263.

Lin HM, Zhao L \& Cheng SY 2002 Cyclin D1 is a ligand-indenpendent co-repressor for thyroid hormone receptors. Journal of Biological Chemistry 277 28733-28741.

Liu WD, Wang HW, Muguira M, Breslin MB \& Lan MS 2006 INSM1 functions as a transcriptional repressor of the NeuroD.beta2 gene through the recruitment of cyclin D1 and histone deacetylase. Biochemical Journal 397 169-177.

Matsuoka T, Zhao L, Artner I, Jarrett HW, Friedman D, Means A \& Stein R 2003 Members of the large Maf transcription family regulate insulin gene transcription in islet beta cells. Molecular and Cellular Biology 23 6049-6062.

Mellitzer G, Bonne S, Luco RF, Van De Casteele M, Lenne N, Collombat P, Mansouri A, Lee J, Lan MS, Pipeleers D et al. 2006 IA-1 is Ngn3dependent and essential for differentiation of the endocrine pancreas. EMBO Journal 25 1344-1352.

Melloul D, Marshak S \& Cerasi E 2002 Regulation of insulin gene transcription. Diabetologia 45 309-326.

Mosley AL, Corbett JA \& Ozcan S 2004 Glucose regulation of insulin gene expression requires the recruitment of $\mathrm{p} 300$ by the b-cell-specific transcripiton factor Pdx-1. Molecular Endocrinology 18 2279-2290.

Naya FJ, Stellrecht CM \& Tsai MJ 1995 Tissue-specific regulation of the insulin gene by a novel basic helix-loop-helix transcription factor. Genes and Development 9 1009-1019.

Naya FJ, Huang HP, Qiu Y, Mutoh H, DeMayo FJ, Leiter AB \& Tsai MJ 1997 Diabetes, defective pancreatic morphogenesis, and abnormal enteroendocrine differentiation in BETA2/NeuroD-deficient mice. Genes and Development 11 2323-2334.

Ohneda K, Ee H \& German MS 2000a Regulation of insuln gene transcription. Seminars in Cell and Developmental Biology 11 227-233.

Ohneda K, Mirmira RG, Wang J, Johnson JD \& German MS 20006 The homeodomain of $\mathrm{Pdx}-1$ mediates multiple protein-protein interactions in the formation of a transcriptional activation complex on the insulin promoter. Molecular and Cellular Biology 20 900-911.
Olbrot M, Rud J, Moss LG \& Sharma A 2002 Identification of beta cell specific insulin gene transcription factor RIPE3b1 as mammalian MafA. PNAS 99 6737-6742.

Prasadan K, Daume E, Preuett B, Spilde T, Bhatia A, Kobayashi H, Hembree M, Manna P \& Gittes GK 2002 Glucagon is required for early insulin-positive differentiation in the developing mouse pancreas. Diabetes 51 3229-3236.

Qiu Y, Guo M, Huang S \& Stein R 2002 Insulin gene transcription is mediated by interactions between the $\mathrm{p} 300$ co-activator and $\mathrm{Pdx}-1$, beta2, and E47. Molecular and Cellular Biology 22 412-420.

Qiu Y, Guo M, Huang S \& Stein R 2004 Acetylation of the beta2 transcription factor by p300-associated factor is important in insulin gene expression. Journal of Biological Chemistry 279 9796-9802.

Ratineau C, Petry MW, Mutoh H \& Leiter AB 2002 Cyclin D1 represses the basic helix-loop-helix transcription factor, beta2/NeuroD. Journal of Biological Chemistry 277 8847-8853.

Shieh SY \& Tsai MJ 1991 Cell-specific and ubiquitous factors are responsible for the enhancer activity of the rat insulin II gene. Journal of Biological Chemistry 266 16708-16714.

Skaper SX, Rhee J, Kim PS, Novitch BG \& Lassar AB 1996 Cyclin-mediated inhibition of muscle gene expression via a mechanism that is independent of pRB hyperhohsphorylation. Molecular and Cellular Biology 16 7043-7053.

Stanojevic V, Habener JF \& Thomas MK 2004 Pancreas duodenum homeobox-1 transcriptional activation requires interactions with p300. Endocrinology 145 2918-2928.

Sussel L, Kalamaras J, Hartigan-O'Connor DJ, Meneses JJ, Pedersen RA, Rubenstein JLR \& German MS 1998 Mice lacking the homeodomain transcription factor $\mathrm{Nkx} 2.2$ have diabetes due to arrested differentiation of pancreatic beta cells. Development 125 2213-2221.

Xie JP, Cai T, Zhang H, Lan MS \& Notkins AL 2002 The zinc-finger transcription factor INSM1 is expressed during embryo development and interacts with the Cbl-associated protein. Genomics 80 54-61.

Zhao L, Guo M, Matsuoka T, Hagman DK, Parazzoli SD, Poitout V \& Stein R 2005 The islet b-cell enriched MafA activator is a key regulator of insulin gene transcription. Journal of Biological Chemistry $28011887-11894$.

Zhu M, Breslin MB \& Lan MS 2002 Expression of a novel zinc-finger cDNA, IA-1, is associated with rat AR42J cells differentiation into insulin-positive cells. Pancreas 24 139-145.

Received in final form 8 April 2008

Accepted 11 April 2008

Made available online as an Accepted Preprint 11 April 2008 